\title{
CROSS-CULTURAL GAME DESIGN: HOW DIFFERENT STUDENTS SEE THE SAME DEVELOPMENT
}

\author{
João Gabriel Guedes Pinheiro and Marco Aurélio Soares dos Santos \\ Universidade do Vale do Itajaí, $5^{a}$ Avenida, 110 - Balneário Camború, SC, Brazil
}

\begin{abstract}
This paper discusses how two groups of students, with different nationalities and academical backgrounds develop the same game. Through literature review, the author profiled the groups and identified five differences on how they work. Results point towards a structured process by bachelor`s students, with master`s taking more liberties in their design process.
\end{abstract}

\section{KEYWORDS}

Game Development, Students, Methods Comparison

\section{INTRODUCTION}

Digital games are a new type of entertainment, even more when compared to cinema or theater: while there are records of Greek plays from the $6^{\text {th }}$ century BCE (Cartwright, 2016), and a predecessor of films from 1872 (Encyclopaedia Britannica, 2018) only 1958 the first digital game was made (APS News, 2008). As games are still new, designers are still building a base of knowledge on game creation, with an example being author who defend a linear approach to game design (Novak, 2010) and others defending an approach based on cycles (Chandler, 2012). These different approaches were observed on a internship opportunity given to bachelor`s students of game design by a company that employs master`s students of game design.

This study is in progress, as the game is being developed, but early results point towards the bachelor`s group having an approach closer to a waterfall method (Hiwiller, 2015) while the master`s group tends more towards the feature driven development (Meravý, 2013). Understanding those differences allows the bachelor`s university to better understand the game development process in an international context, while guiding students towards more effective methods of game design.

\section{METHODOLOGY}

This work is based on a case study, as proposed in (Liem, 2017), of a game in development by master`s students, that the group of bachelor`s students joined in a later date. To perform the analysis, the authors utilized as reference for methods adopted by both groups in bibliography cited by the two game development programs, while also utilizing authors that refers to methods used by the teams, but not found in their references: an example of this is (Goodwin, 2016), that while not used as a reference in the design programs analyzed, discuss version control, a system used during the project development.

\section{DEVELOPMENT CULTURES}

It's undeniable that the north-American game industry is a reference for Brazilian game developers: according to (Gamedevmap, 2019), United States has 1634 game companies, developing areas varying from serious games to mobile. Brazil is in a different stage: while it's going through a growth in number of game companies of 164\%, (Wakka, 2018), the number of formal game development studios is still 276. 
To understand if there is a difference between how game development is thought, the authors used an ongoing project that involves master's students from an american university, and bachelor's students from a brazilian university. Although there could be an innate difference in how the two groups operate through their previous experiences, not every master's student have a background on game development, and some of the bachelor's students had it. It's also important to comprehend that as bibliographies in universities programs doesn't vary too much throughout time, they can be used to make a case for their practices being similar to how games are made within those cultures.

This study uses a table, based on literature review, to identify some of the differences between the two groups of students. As the game is still in development, and more features are expected to be implemented, it`s not possible to emphasize all the differences observed between both teams. Instead, the authors focus on five elements observed during development of most games, from subjects of how they create their visions, to how they approach the game documentation and their views on playtesting. Only in the future, after the game is complete, a more detailed analysis can be made on which of those two different views on game design provided the best results, or had a better use of resources to achieve the results desired.

\subsection{On their Practices}

As (Brathwaite \& Schreiber, 2009) discusses different approaches in game design, this view was adopted to identify how, from a creative view, their games are made and their different priorities. In the groups observed in this study, two main views were observed, the blue-sky and the mechanic-driven design. The first approach is more creative when compared to the second, where the designers allow themselves to pursues different ideas, ignoring constraints such as time or resources. For a mechanic-driven design, the game is made around one idea, with the remaining aspects following it. So if a game is focused on the idea of racing, every mechanic and system will be build to better deliver the experience of racing.

About documentation, (Novak, 2010) discusses the use of a single design document, that contains every material needed for designers, artists and developers to create their game. While this view is more common within the industry, (Adams, 2013) comments on the use of different documents that fulfill different needs. This analysis can be used to not only identify the best way to introduce a new team member to a project, but also how to keep track of development: a centralized document may make it easier to understand the project, as it contains the entire process, whle different documents can provide a better comprehension of different aspects of the game.

As games are made of features, it's also valid to identify the main tool in which those features were thought of, as there are methods that can use existing games as a reference, with SCAMPER being an example of it (Brynteson, 2012), to creating user stories that define what the user would like to do, and then creating a system to assure him that experience (Maioli, 2018). This difference can also be used to exemplify how different their processes are when making a game: SCAMPER is better used with an existing design, where you reorganize their elements in order to improve it, while user stories doesn't depend on existing systems to be used.

Comparing how each team uses version control can also explain their views on the process, as working on the main branch only may indicate some lack of concern with stability of the build, as some of the work that's done may cause a negative impact on the game (as creating major bugs), while worrying about side branches may indicate that the team is concerned to not affect the main branch if some asset creates unexpected results (Goodwin, 2016).

The last comparison is between tests: playtesting throughout the development is a practice encouraged, as it allows the team to understand how the game is played, and where it is when compared to their goals (Kramarzewski \& De Nucci, 2018). This can be used to measure how progress is being tracked: if the game is tested frequently, it can be assumed that the team has a better idea of where the game is in its pipeline, while not testing may indicate possible issues on its quality control.

\subsection{Comparison}

The Table 1 contains a comparison between the groups of students, based on the criteria specified previously. It can be noted that they have major differences in how they think of features in their games: the Master`s group tends to think more towards the user experience, while losing some structure in the process, that may create 
obstacles along the development, from levels being added to the build without being tested to the usage of outdated documents in the game.

The Bachelor`s group, instead, follows a more rigid structure: instead of only focusing on the user experience, there`s a bigger concern with the formality of the design, through the use of a single document, the use of different branches as was asked by the Master`s group, to the use of tests to identify problems before they are added to the main branch of the game. Such a view does present a concern, that their view of the game is not open to expansions when compared to the Master`s group, as their system is more defined, and for that reason less open to the creation of new features, or changes to existing ones.

Table 1. Comparison of the groups

\begin{tabular}{lll}
\hline & Master`s & Bachelor`s \\
\hline Game design approach & Blue-sky & Mechanic \\
Documentation & Different documents & A single document \\
Methods for concept creatic User Stories & SCAMPER \\
Version control & Main branch & Different branches \\
Testing & Not usual & Frequent tests \\
\hline
\end{tabular}

\section{CONCLUSION}

This study attempts to profile groups of students, from different nationalities and academical experiences, to better understand how games are made, and how bachelor`s students can experience a different view on game design, from developers with more experience in it. Through literature review, and the use a game in which bachelor`s students joined a project in development by master`s students as a case study, the authors profiled both groups using concepts of game creation, allowing them to understand the differences between the two groups, and how the bachelor`s education can be adapted to better fit the industry expectations.

\section{ACKNOWLEDGEMENT}

The authors are grateful for the support of Zygobot, that kindly gave the team of students from Univali an opportunity to work in their game, allowing the authors to start this study. They are also grateful for Univali allowing students to take part in such an opportunity, and offering its space and resources.

\section{REFERENCES}

Adams, E., 2013. Fundamentals of Game Design. 3 ed. s.1.:New Riders.

APS News, 2008. October 1958: Physicist Invents First Video Game. [Online] Available at: https://www.aps.org/publications/apsnews/200810/physicshistory.cfm [Accessed 3 January 2019].

Brathwaite, B. \& Schreiber, I., 2009. Challenges for Game Designers. Boston: Cengage Learning.

Brynteson, R., 2012. Innovation at Work. s.1.:Amacom.

Cartwright, M., 2016. Ancient Greek Theatre. [Online] Available at: https://www.ancient.eu/Greek_Theatre/ [Accessed 3 January 2019].

Chandler, H. M., 2012. Manual de producao de jogos digitais [The game production handbook]. Porto Alegre: Bookman.

Encyclopaedia Britannica, 2018. Eadweard Muybridge. [Online] Available at: https://www.britannica.com/biography/Eadweard-Muybridge [Accessed 2 January 2019].

Gamedevmap, 2019. Gamedevmap. [Online] Available at: https://www.gamedevmap.com/index.php?country=United\%20States\&state=\&city=\&query=\&type= [Accessed 16 March 2019].

Goodwin, S., 2016. Polished Game Development: From First Steps to Final Release. London: Apress.

Hiwiller, Z., 2015. Players Making Decisions: Game Design Essentials and the Art of Understanding Your Players. s.1.:New Riders. 
Kramarzewski, A. \& De Nucci, E., 2018. Practical Game Design. s.1.:Packt Publishing. Liem, A., 2017. Prospective Ergonomics. London: Wiley-ISTE.

Maioli, L., 2018. Fixing Bad UX Designs. s.l.:Packt Publishing.

Meravý, J., 2013. Effective Leadership in Game Industry using Agile Methods. Brno: s.n.

Novak, J., 2010. Desenvolvimento de games [Game development essentials]. Sao Paulo: Cengage Learning.

Ratcliffe, L. \& McNeill, M., 2011. Agile Experience Design: A digital designer's guide to agile, lean, and continuous. Berkeley: New Riders.

Scolastici, C. \& Nolte, D., 2013. Mobile Game Design Essentials. Birmingham: Packt Publishing.

Wakka, W., 2018. Cresce número de empresas de jogos no Brasil, mas salário ainda é baixo. [Online] Available at: https://canaltech.com.br/games/cresce-numero-de-empresas-de-jogos-no-brasil-mas-salario-ainda-e-baixo-126545/ [Accessed 16 March 2019]. 